\title{
A note on price volatility in the Australian electricity market
}

\author{
C. Conticini ${ }^{1} \quad$ J. A. Filar ${ }^{2} \quad$ A. Nazari ${ }^{3}$
}

(Received 20 June 2010; revised 22 September 2010)

\begin{abstract}
Efficiency in electricity transmission has been a worldwide 'hot topic' for more than ten years and has led to reforms in many countries. However, the performance of the restructured electricity markets is often questioned since it depends, to a large extent, on the way market participants collectively respond to market rules and procedures. We examine data from Australia's National Electricity Market with emphasis on variability in spot prices. These data indicate that the structure of electricity generators' bidding offers may be one of the important contributors to the volatility in electricity prices. A scheme for reducing the volatility of spot prices is then proposed in the form of new regulations on the structure of generators' electricity price and volume bids.
\end{abstract}

http://anziamj . austms.org.au/ojs/index.php/ANZIAMJ/article/view/2655 gives this article, (C) Austral. Mathematical Soc. 2010. Published October 4, 2010. ISSN 1446-8735. (Print two pages per sheet of paper.) Copies of this article must not be made otherwise available on the internet; instead link directly to this URL for this article. 


\section{Contents}

1 Introduction

C731

1.1 Behaviour of demand and price . . . . . . . . . C733

1.2 The volatility . . . . . . . . . . . . . . C C734

2 Mathematical formulation

C736

2.1 Notation for mathematical formulation . . . . . . . . C737

2.2 The problems and new scheme for pricing . . . . . . C738

3 Numerical results

C742

4 Conclusion

C743

References

C744

\section{Introduction}

Since the deregulation in the early 1990s the Australian electricity market has operated under a specially structured competitive regime. In the progress from a regulated monopoly to a deregulated market, the pricing structure was said to have been completely reformed according to a principle of optimising the system and providing significant saving to end-users [1]. However, it has been observed that electricity prices in the deregulated market are characterised by a volatility that varies over time and occasionally reaches extremely high levels. Apparently, there is no consensus as to whether this volatility is a direct, or indirect, consequence of the market structures, or whether it is primarily due to exogenous factors such as the weather and demand fluctuations. However, understanding the volatility process is critically important to distributors, generators and market regulators so as to allow them to better manage their financial risks.

The National Electricity Market (NEM) is the centre of the Australian dereg- 
ulated electricity industry. It is a spot market in which demand and supply are matched on a five minute basis and prices are determined. Half-hour averages are used for commercial transactions. This market is believed to offer an effective design for stimulating competition among electricity generators. However, the need to monitor and improve market rules is well recognised, and refinements to the design may be expected in future especially with increasing wind farm components [9].

The electricity demand or load are highly volatile in a manner that depends on time of the day, day of the week, temperature and other parameters. During a typical day, peak loads occur from 7:00 am to 9:00 am and 4:00 pm to 7:00 pm. Also, load is highly correlated to the temperature, especially with the increasing usage of air conditioners during summer and heating devices during winter. In addition, the electricity market exhibits extreme situations when the generators reach their maximum capacity, but there is still more demand. The excess demand can be supplied at prices many times higher than the normal price level [5].

The literature on electricity pricing and volatility is broad and generally categorised in two groups [5]. The first group consists of statistical and mathematical modelling of the stochastic properties of prices [10, e.g.]. The second group utilises economic equilibrium models of the supply and demand functions for pricing [8, e.g.].

The volatility of the electricity price could originate from a number of sources. We discuss the impact of generators' bids on the price volatility and accompanying spikes. To reduce this kind of volatility, we propose a new price bidding scheme which decreases the volatility and at the same time has a positive impact on the reduction of power transmission and power loss. Our aim is to propose a scheme to decrease overall energy cost for end-users. This is because, thanks to hedge contracts between generators, retailers and large consumers, generators are substantially insulated from the risks of price volatility [11]. 


\subsection{Behaviour of demand and price}

Ostensibly, the electricity market design stimulates competition among generators so that the total behaviour of the system is optimised. However, it appears this market design also encourages highly irregular bid offers (for example, see Figure 1). For instance, there is disparity among bands 6, 7 and 8 both in terms of price and volume. ${ }^{1}$ Could these disparities significantly contribute to the price volatility?

Generally, the price is relatively stable at between $\$ 10$ and $\$ 80$ per MWh. The Winter average price (around \$35) is lower than the Summer average price (around \$55). The main reason for the high prices in Summer is the use of air conditioners by households and industry. All year long, small peak prices occur around 7:00 am to 9:00 am and 4:00 pm to 7:00 pm, times of the day when the electricity demand is high. However, during some weeks - almost exclusively in Summer - sharp peaks approaching the maximum price of $\$ 10,000$ occur not so infrequently. For example, on the 18th of February 2008, the price reached $\$ 10,000$ from 1:00 pm to $6: 30 \mathrm{pm}$, as shown in Figure 2. Note that, from the corresponding demand curve (in the lower panel of Figure 2) the demand, though a little higher than on other days, did not increase nearly as sharply as the price. According to AEMo (Australian Energy Market Operator), high prices are used as a protection against high demand, since it can be harmful for a generator to produce electricity at full power for a long time. When predicting high demand, generators would then use extreme prices to discourage consumers from further use of electricity. However, AEMO has other means to decrease the demand and does not hesitate to use them. For example, when the demand is too high in a region, electricity may be imported from nearby regions through interconnectors. Moreover, some consumers (mostly companies) are willing, for a financial consideration, to decrease their electricity demand at times of supply shortfalls, and AEMO has the power to temporarily suspend the supply of customers in a specific part

${ }^{1}$ Arguably, it is counterintuitive that a generator willing to produce $100 \mathrm{MW}$ at a price of $\$ 150$ is unwilling to produce any at price of $\$ 3,700$. 


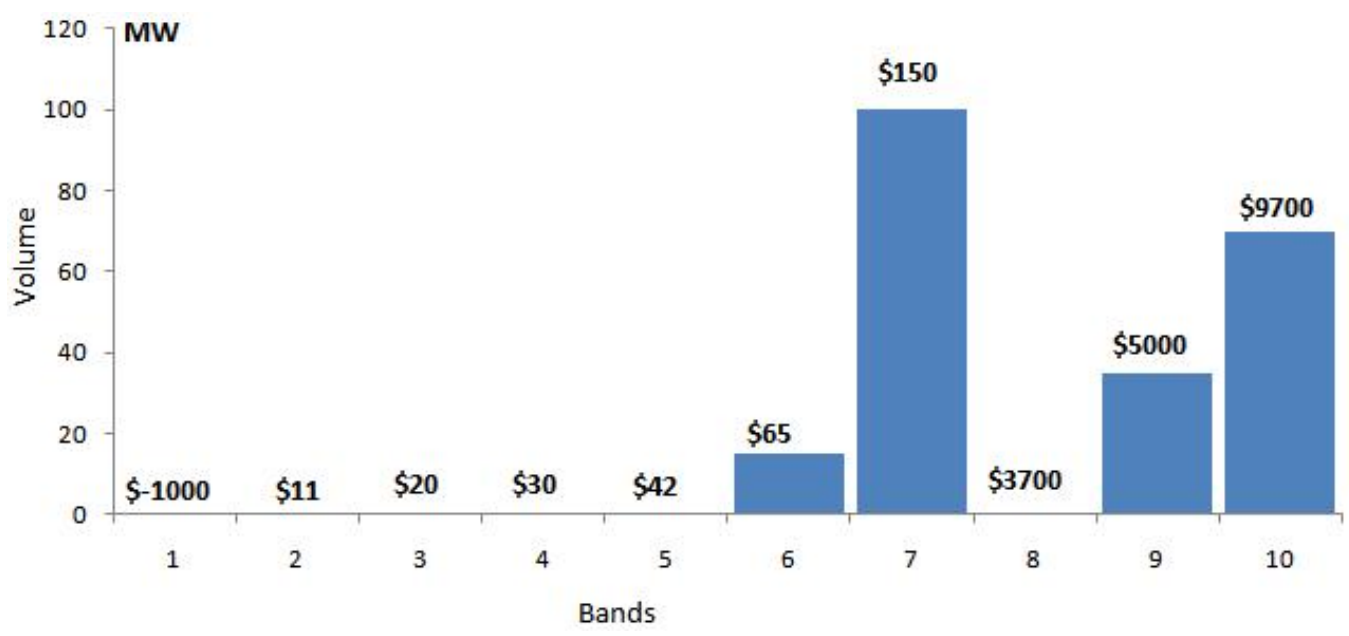

Figure 1: A typical bid offer containing ten bands of volume and related prices.

or region of the National Electricity Market, if such an extreme action were deemed necessary [3].

\subsection{The volatility}

Volatility in spot prices is an important factor that influences a degree of uncertainty at what the spot price might be during any given period. At times when demand exceeds the generation, the electricity price can jump to the cap price of $\$ 10,000$ per MWh. Many factors may have positive or negative impact on the volatility of spot prices including market structure and market power [6]. However, a high level of extra capacity is built into the operations of the current system. Hence, the observed phenomenon of price spikes hitting the cap price suggests the presence of other causes for volatility beyond the simple corollary of the supply-demand principle [4].

The objective of this article is to demonstrate the presence of the volatility 


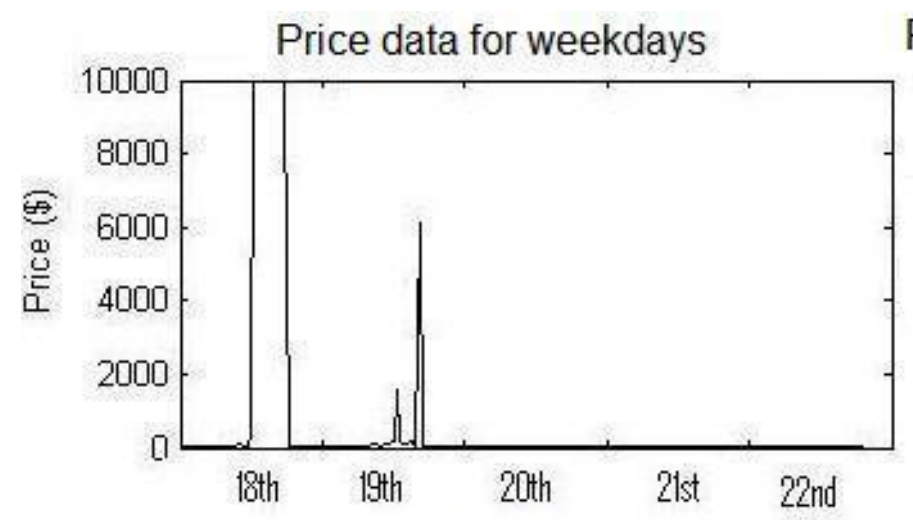

Price data for weekend

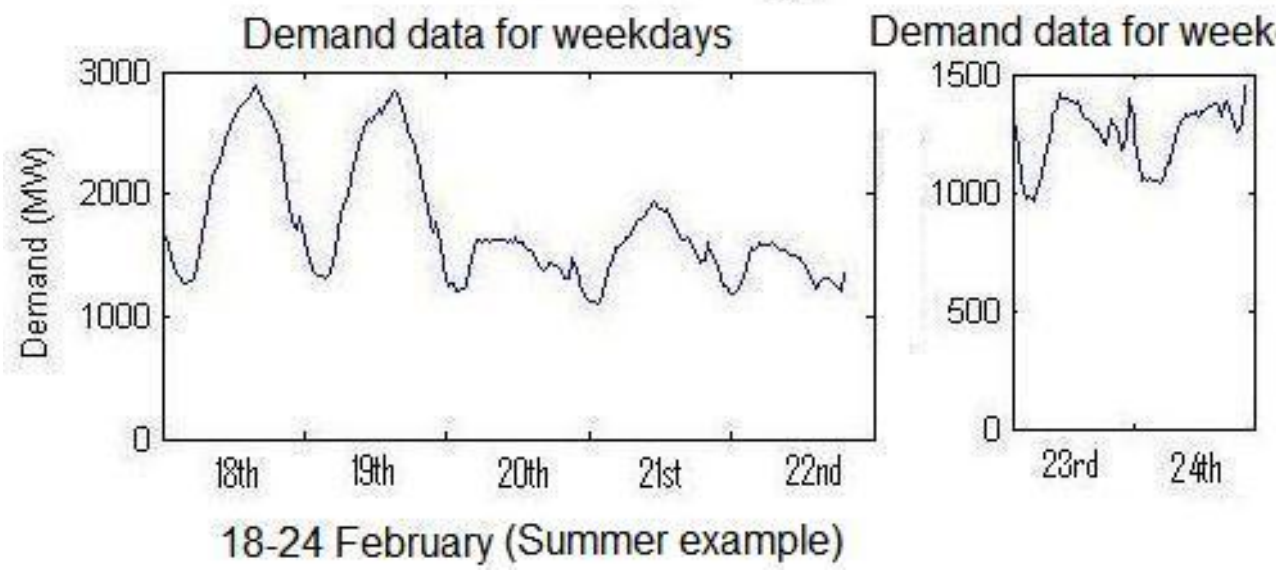

Figure 2: Typical price and demand curves during Summer

TABLE 1: Standard deviation, mean and coefficient of variation.

\begin{tabular}{c|ccc|ccc|}
\multirow{2}{*}{} & \multicolumn{3}{|c}{ 16th to 22nd June } & \multicolumn{4}{c}{ 18th to 24th February } \\
\cline { 2 - 7 } & $\sigma_{\mathrm{J}}$ & $\mu_{\mathrm{J}}$ & $\mathrm{c}_{\mathrm{J}}$ & $\sigma_{\mathrm{F}}$ & $\mu_{\mathrm{F}}$ & $\mathrm{c}_{\mathrm{F}}$ \\
\hline Demand & 254 & 1600 & 0.16 & 471 & 1597 & 0.29 \\
Price & 15 & 36 & 0.41 & 1879 & 412 & 4.55 \\
\hline
\end{tabular}


component of the spot price stemming from generators' bidding structure (see Figure 1 for a typical example). To the extent that these structures reflect the mechanism by which spot prices are calculated, this volatility component is 'man-made' and well suited possible for reductions.

Definition 1 For a data set $\mathrm{X}=\left\{x_{1}, \ldots, x_{n}\right\}$, the coefficient of variation is $\mathrm{c}=\sigma / \mu$, where $\sigma$ and $\mu$ are the sample standard deviation and the sample mean of data set $\mathrm{X}$.

The coefficient of variation is a widely used index for measuring the volatility [2] that removes scale effects. If the coefficient is small relative to one, then the variable has low volatility; conversely, if the coefficient is close to or greater than one, then the variable is highly volatile. In order to compare the volatility of price and demand, the standard deviation and coefficient of variance are calculated for the periods 16th to 22nd of June and 18th to 24th of February in 2008 (see Table 1). The discrepancy in c values is obvious, especially for data from 18th to 24th of February in 2008 which is striking. Note that for the demand variable the coefficient of variation increased by a factor of less than two while for the price variable it increased by a factor of more than ten.

\section{Mathematical formulation}

The mathematical description of the problem is presented in three parts. The dispatch problem which is a simplified electricity purchase optimization problem and is conceptually similar to the actual problem faced by AEMO. An analogous utopian problem (electricity production and dispatch together) is solved, as a benchmark, when the objective is the benefit of consumers. It is a nonlinear optimization problem where both the volume and the price for each band are decision variables. An optimal solution of the latter demonstrates feasible, if idealistic, energy bids with the lowest possible cost. Finally, we propose a solution to achieve more stable spot prices by introducing a new 


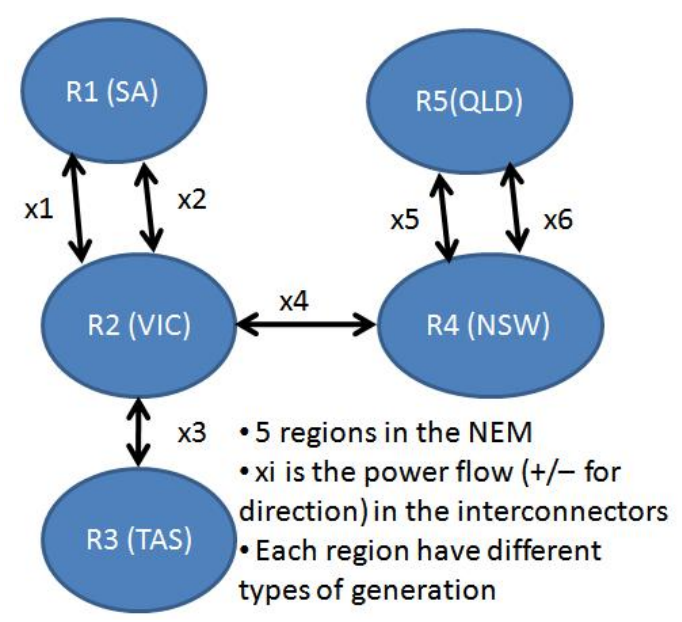

FiguRE 3: Nem electricity grid

structure for generators' electricity bids.

\subsection{Notation for mathematical formulation}

We consider the electricity grid to be a connected graph whose nodes are numbered from R1 to R5 and correspond to five Australian eastern states, as shown in Figure 3. The interconnectors are numbered from 1 to 6 , and the flow on these lines is denoted by variables $x_{1}$ to $x_{6}$. In the real grid, electricity can be transmitted through the interconnectors in both directions, as shown in Figure 3. That is why in this problem the $x_{i}$ variables can be negative. In the following mathematical formulation, the generators are indexed by $\mathrm{g} \in \mathrm{G}=\left\{1,2, \ldots, \mathrm{n}_{\mathrm{G}}\right\}$, where $\boldsymbol{n}_{\mathrm{G}}$ is the number of generators considered in the problem, and the bands are indexed by $b \in B=\{1, \ldots, 10\}$ (Table 2 gives further notation). 
TABLE 2: Notation.

\begin{tabular}{ll}
\hline Symbol & Definition \\
\hline$c_{b}^{g}$ & price offered by the generator $g$ for the bth band \\
$y_{b}^{g}$ & volume offered by the generator $g$ for the bth band \\
$z_{b}^{g}$ & volume of electricity purchased from the $b$ th band of \\
& generator $g$ \\
maxw $_{i}$ & maximum flow in interconnector with flow $x_{i}$ \\
minw $_{i}$ & minimum flow in interconnector with flow $x_{i}$ \\
$d_{j}$ & demand at the region $j$ \\
$\mathrm{mg}^{g}$ & maximum capacity of generator $g$ \\
$\mathrm{mb}_{\mathrm{b}}^{\mathrm{g}}$ & maximum volume band can be offered $\left(\max _{\mathrm{b}=1, \ldots, 10}\left\{\mathrm{y}_{\mathrm{b}}^{\mathrm{g}}\right\}\right)$ \\
$\mathrm{mp}$ & minimum price for the second to tenth bands \\
$\mathrm{ms}$ & minimum step between the price of two consecutive bands \\
\hline
\end{tabular}

\subsection{The problems and new scheme for pricing}

The operation of the NEM is underpinned by a sophisticated information technology system. The core of this system is the dispatch problem which consists of a linear programming problem. Generators' offers to supply a specific amount of electricity at a particular price are the main information provided to the dispatch system beside some other network related and technical considerations. From all offers, which are submitted every five minutes, the solution of the linear programming dispatch problem selects the generators required to produce electricity, ostensibly based on the principle of meeting prevailing demand in a most cost effective manner. However, each generator $g$ is permitted to choose the price $c_{b}^{g}$ and amount $y_{b}^{g}$ of electricity offered at that price for $\mathrm{b}=1,2, \ldots, 10$ corresponding to the ten price bands. The quantity of electricity which is going to be purchased from each band of each generator is decided by AEMO and is limited by the maximum volume the generators are willing to produce. Therefore, the dispatch problem is a linear programming problem where the decision variables are denoted by $z_{\mathrm{b}}^{\mathrm{g}}$, as in Table 2. In this dispatch problem, the solution is an array containing 
optimal dual variables values ("shadow prices") corresponding to the five demand constraints in (1). The latter become the five spot prices (one for each of the five NEM regions) paid by AEMO, for all the electricity purchased for these regions. By solving the dispatch problem, we are also solving an optimal power flow problem [7].

$$
\begin{array}{ll}
\min _{(g, b) \in G \times B} c_{b}^{g} z_{b}^{g} \quad \text { such that } & \\
\sum_{g \in R_{j}, b \in B} z_{b}^{g}-\sum_{i \in E \times p^{j}} x_{i}+\sum_{i \in \operatorname{Imp}^{j}} x_{i}=d_{j} & \text { for all } j \in\{1, \ldots, 5\} \\
0 \leqslant z_{b}^{g} \leqslant y_{b}^{g} & \text { for all } g \in G, b \in B \\
\operatorname{minw}_{i} \leqslant x_{i} \leqslant \operatorname{maxw}_{i} & \text { for all } i \in\{1, \ldots, 6\} .
\end{array}
$$

Remark 2 It is crucially important to note that since the spot prices paid for all the electricity purchased are optimal values of the dual variables of (1), they are functions of the $\left(\mathrm{c}_{\mathrm{b}}^{g}, \mathrm{y}_{\mathrm{b}}^{g}\right)$ parameters supplied by the generators. If the simplex method is used to solve (1), then this dependence will in general exhibit complex behaviours because even small changes in these parameters may cause a change in an optimal basis.

The utopian problem is a nonlinear optimisation problem in which both the price and the volume for each band are variables. The goal is to minimise the cost of the electricity purchased while meeting the demands in all five regions of NEM. In this approach, the only goal of the system is the benefit to the consumers.

$$
\begin{array}{ll}
\min _{(g, b) \in G \times B} c_{b_{b}^{g}}^{g} \text { such that } & \\
\sum_{g \in R_{j}, b \in B} z_{b}^{g}-\sum_{i \in E \times p^{j}} x_{i}+\sum_{i \in I_{p} p^{j}} x_{i}=d_{j} & \text { for all } j \in\{1, \ldots, 5\} \\
c_{b}^{g} \leqslant c_{b+1}^{g}-m s & \text { for all } g \in G, b \in B \backslash\{10\} \\
-1000 \leqslant c_{1}^{g} & \text { for all } g \in G \\
\operatorname{mp} \leqslant c_{b}^{g} \leqslant 10000 & \text { for all } g \in G, b \in B \backslash\{1\}
\end{array}
$$




$$
\begin{array}{ll}
1.05 z_{b}^{g} \leqslant z_{b+1}^{g} & \text { for all } g \in G, b \in B \\
0.01 \mathrm{mg}^{g} \leqslant z_{b}^{g} \leqslant \operatorname{mb}_{b}^{g} & \text { for all } g \in G, b \in B \\
\text { minw }_{i} \leqslant x_{i} \leqslant \operatorname{maxw}_{i} & \text { for all } i \in\{1, \ldots, 6\}
\end{array}
$$

The scheme we present next consists of imposing additional regulations on generators when creating their offers that is meant to reduce the volatility in spot price. The ten bands principle will be respected, permitting AEMO to retain its purchase optimisation algorithm (1).

Figure 1 shows that there are, potentially, huge jumps in generators' pricedemand bids. The idea here is to prevent such jumps by requiring generators to construct their bids from an appropriate discretisation of a smooth, monotone, function $p(v)$ that they can tailor to their own requirements. The function $p(v)=\alpha v^{\mathfrak{n}}+p_{s}$ represents price as a function of volume $v$, where $p(0)=p_{s}$ denotes the start price that is bounded below by the currently used gift price of $-\$ 1,000 / \mathrm{MWh}$ and $\mathrm{p}(\mathrm{mg}) \leqslant \$ 10,000$. The generators are free to choose the parameters $\alpha, n$ and $p_{s}$ of the function $p(v)$. However, once they choose these parameters, they would be required to create their ten band price-volume bids by the following discretization scheme. For a given generator, $g$, let $v_{\mathrm{b}}:=z_{\mathrm{b}}^{\mathrm{g}}$ from the optimal solution of the utopian problem for $b=1, \ldots, 10$, and $v_{0}=0$. Then, the discrete price, in the band $b$, is

$$
c_{b}=\frac{1}{v_{b}-v_{b-1}} \int_{v_{b-1}}^{v_{b}} p(v) d v, \quad \text { for all } b \in B .
$$

This scheme imposes a form of regularity in the bid volumes that is a consequence of the monotonicity constraints $1.05 z_{\mathrm{b}}^{\mathrm{g}} \leqslant z_{\mathrm{b}+1}^{\mathrm{g}}$, for all $\mathrm{g} \in \mathrm{G}$, $\mathrm{b} \in \mathrm{B}$ in (2); see Figure 4. Zero bid volumes no longer arise due to constraints $0.01 \mathrm{mg}^{\mathrm{g}} \leqslant z_{\mathrm{b}}^{\mathrm{g}} \leqslant \mathrm{mb}_{\mathrm{b}}^{\mathrm{g}}$, for all $\mathrm{g} \in \mathrm{G}, \mathrm{b} \in \mathrm{B}$ in (2). One important implication of the scheme is that there are no longer zero production gaps, at a positive price, in the generators' bids. That is, if a generator is willing to produce a positive amount of electricity at one price he must also be willing to produce at least as much at every higher price. As a consequence, generators 

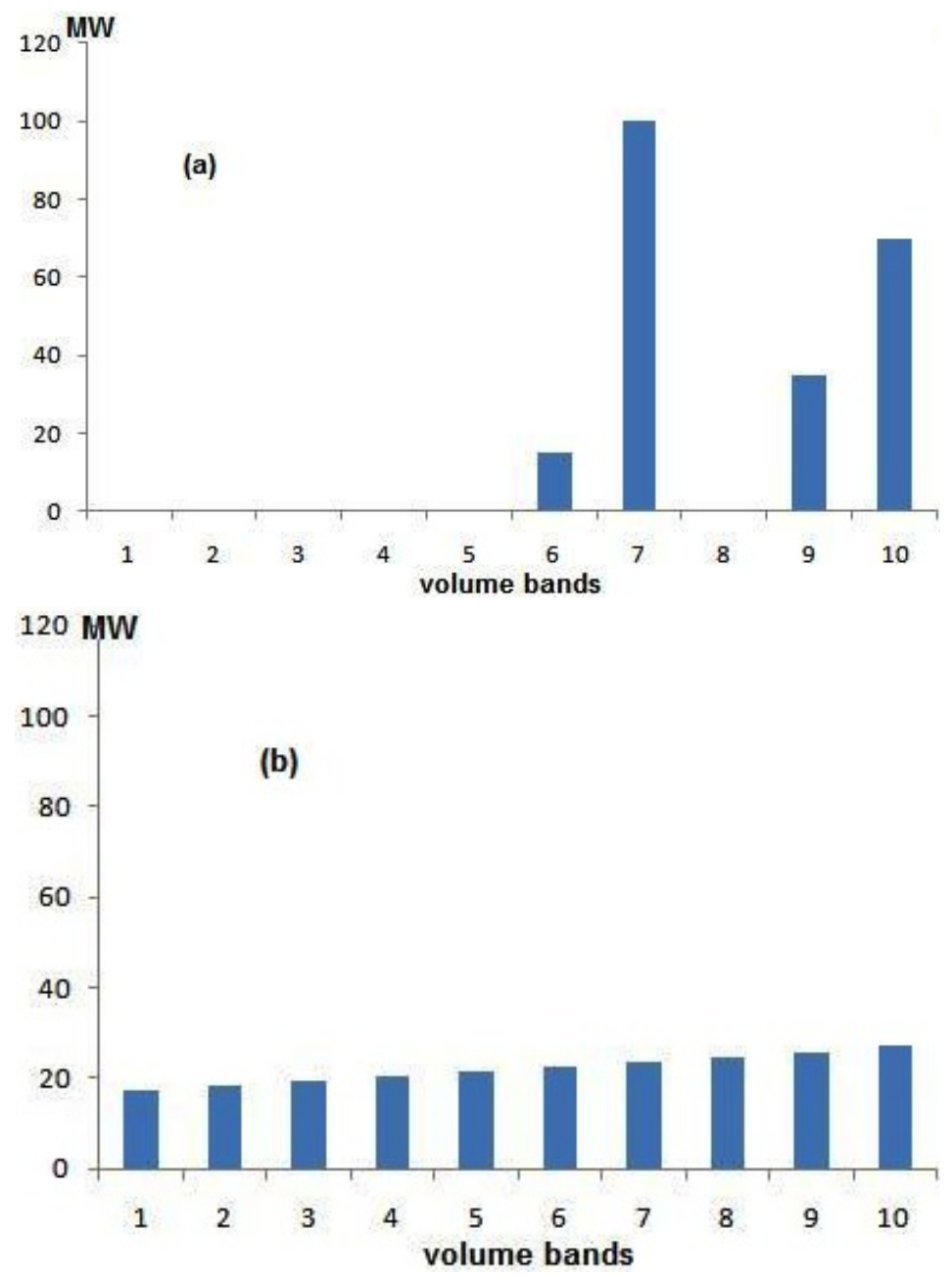

Figure 4: Typically, continuity regulation converts bid volume stacks from (a) into (b). 
are prevented from increasing the price of electricity without a commitment to producing it. However, in order not to unduly disadvantage generators we shall assume that the parameters of the $p(v)$ function are chosen to satisfy $\int_{0}^{C a p} p(v) d v=I$, where Cap denotes the total production capacity of the generator and I denotes the total income received if the entire bid offer of that generator were purchased. There is some flexibility in the choice of parameters of the $p(v)$ function that satisfy the preceding requirement. In particular, the question of whether $n$ should be smaller or greater than one is related to whether there are economies or diseconomies of scale in electricity production.

\section{$3 \quad$ Numerical results}

This section demonstrates the effect of the proposed new scheme on spot prices volatility and power loss. We compare the solution of the actual dispatch problem (used by AEMO) and a solution resulting from the new pricing scheme.

To do this we derive a data set of synthetic spot prices $S_{t}$ resulting from the proposed new scheme, for each trading period in January 2010. These data will be the base of calculations for descriptive statistics: the average, the standard deviation and the coefficient of variation. Table 3 reports these statistics for both real data and synthetic data.

The results show that by creating new bidding regulations imposing a degree of regularity on the pricing scheme and volumes, it is possible to decrease the spot price volatility, and maybe even the overall electricity cost. Note that, for actual data, the coefficient of variation in spot price for January 2010 was 7.76 reflecting very high volatility. However, under the proposed new scheme it dropped to only 0.51. This constitutes a considerable improvement. The spot prices obtained by the new scheme are sometimes higher than those obtained in the utopian problem, but are generally lower than those obtained 
TABLE 3: Descriptive statistics for demand, actual and synthetic spot prices for January 2010.

\begin{tabular}{lccc} 
& $\sigma$ & $\mu$ & $\mathrm{c}$ \\
\hline Demand & 445.8 & 1584 & 0.28 \\
Old Price & 755.8 & 97.3 & 7.76 \\
New Price & 18.32 & 35.8 & 0.51 \\
\hline
\end{tabular}

in the actual dispatch problem.

For the purpose of illustration, the parameters $n$ and $p_{s}$ in the function $p(v)$ were set to one and zero, respectively, and $\alpha$ was chosen so that the total income bid by generators is the same as in the original pricing structure. However, the new pricing scheme offers substantial flexibility in the choice of the price curve parameters and there are many ways for the generators to create offers leading to spot prices that are more stable.

Another important aspect of solving the utopian problem first and then entering the results into the dispatch problem combined with a pricing scheme, is seen in the interconnector flows. Generally, power loss in the network is one of the important issues in energy transmission that wastes almost $10 \%$ of the generated electricity. Also, in a real situation, part of the price paid by customers and end-users is due to the network services. Therefore, with high interconnector transmission, there will be higher price for electricity. Comparing results in the Table 4, it is apparent that the amount of electricity transmission in the proposed new scheme is almost half of the transmission level in the dispatch solution or the utopian solution.

\section{Conclusion}

We have discussed the observed phenomenon of high volatility of spot prices in the Australian electricity market. We conjectured that the highly irregular 
TABLE 4: Electricity flow in the interconnectors ( \pm signs indicate the flow direction).

\begin{tabular}{lrrrrrrl}
\hline Power Flow & $\chi^{1}$ & $\chi^{2}$ & $\chi^{3}$ & $\chi^{4}$ & $\chi^{5}$ & $\chi^{6}$ & $\begin{array}{l}\text { Total trans- } \\
\text { mission }\end{array}$ \\
\hline Dispatch (MW) & 460 & 220 & -488 & 1592 & -1080 & -180 & 4020 \\
Proposed (MW) & -70 & 59 & -600 & 836 & -783 & 1 & 2349 \\
\hline
\end{tabular}

form of bid offers from generators is a contributing factor to this phenomenon. To decrease the volatility, we propose a new pricing scheme that imposes some regularity on the pricing function and volume of bids. To minimise adverse impact on generators, the parameters of the pricing function are chosen so that they could, in principle, earn the same amount under the new pricing structure. With synthetic data we demonstrate that this approach can, indeed, reduce volatility and decrease the power flow in the interconnectors between states. The latter leads to a desirable reduction in power loss and hence, ultimately, to lower electricity cost for customers and end-users.

Acknowledgements Supported by the Australian Research Council grant (DP0987148) and Linkage grant (LP0884005). Thanks to Assoc. Prof. John Boland from UNISA and Dr. David Swift from AEMO.

\section{References}

[1] Australian Bureau of Statistics: Year Book Australia, Energy: Special Article - Reforms in the Australian Electricity and Gas Industries, http: //www.abs.gov.au/ausstats/abs@.nsf/featurearticlesbytitle/ C50CABC70C99E249CA2569E3001FF8A5?OpenDocument C731

[2] A. Kay-Spratley, A. Worthington and H. Higgs, Transmission of price and price volatility in australian electricity spot market: a multivariance 
garch analysis. Energy Economics, 27:337(350), 2005.

doi:10.1016/j.eneco.2003.11.002 C736

[3] An introduction to Australia's National Electricity Market, July 2009, http://www.aemo.com.au/corporate/0000-0262.pdf C734

[4] D. Brennan and J. Melanie, Market power in the autralian power market, Energy Economics, 20:121(133), 1998. doi:10.1016/S0140-9883(97)00010-8 C734

[5] M. Goto and G. Karolyi, Understanding Electricity Price Volatility within and across Markets, Working Paper Series 2004-12,2004. http://econpapers.repec.org/RePEc:ecl:ohidic:2004-12 C732

[6] H. Higgs, Price and volatility relationships in the Australian electricity market, PhD Thesis, submitted 2006, Queensland University of Technology. http://eprints.qut.edu.au/16404/ C734

[7] D. P. Kothari and I. J. Nagrath, Modern Power System Analysis, McGraw-Hill, 2008. ISBN 978-0-07-340455-4 C739

[8] F. Longstaff and A. Wang, Electricity Forward Prices: A High-Frequency Empirical Analysis, Journal of Finance, Vol. 59(4) Aug. 2004, pp.1877-1900. doi:10.1111/j.1540-6261.2004.00682.x C732

[9] H. Outhred, The competitive market for electricity in Australia: why it works so well, Proceedings of the 13rd International Conference on System Sciences, 2000. doi:10.1109/HICSS.2000.926760 C732

[10] S. Stoft, Power System Economics, John Wiley, New York, NY, 2002. http://au.wiley.com/WileyCDA/WileyTitle/ productCd-0471150401.html C732

[11] F. A. Wolak, An empirical analysis of the impact of hedge contracts on bidding behavior in a competitive electricity market, Stanford University, 1999. doi:10.1080/10168730000000017 C732 


\section{Author addresses}

1. C. Conticini, Ecole Nationale Superieure des Techniques Avances, French Engineering School, 32 Boulevard Victor, 75015 Paris, FRAncE. mailto: celia.conticini@ensta.fr

2. J. A. Filar, Institute for Sustainable Systems and Technologies, School of Mathematics and Statistics, The University of South Australia, Mawson Lakes, SA 5095, Australia. mailto: jerzy.filar@unisa.edu.au

3. A. Nazari, Institute for Sustainable Systems and Technologies, School of Mathematics and Statistics, The University of South Australia, Mawson Lakes, SA 5095, Australia.

mailto:asef .nazari@unisa.edu.au 